LBNL-43572

SC-MAG-674

\title{
REFRIGERATION OPTIONS FOR THE ADVANCED LIGHT SOURCE SUPERBEND DIPOLE MAGNETS
}

\author{
M. A. Green, E. H. Hoyer, R. D. Schlueter, C. E. Taylor and J. Zbasnik \\ E. O. Lawrence Berkeley National Laboratory \\ University of California \\ Berkeley, CA 94720
}

S. T. Wang

Wang NMR Incorporated Livermore CA 94550, USA

\section{Cryogenic Engineering Conference \\ Montreal, Quebec, Canada \\ July 13 through July 16,1999}

To be published in Advances in Cryogenic Engineering, Vol. 45 (1999)

*This work was performed at the Lawrence Berkeley Laboratory with the support of the Director, Office of Energy Research, Office of High Energy and Nuclear Physics, High Energy Physics Division, U. S. Department of Energy under Contract No. DE-AC03-76SF00098. 
LBNL-43572

\title{
REFRIGERATION OPTIONS FOR THE ADVANCED LIGHT SOURCE SUPERBEND DIPOLE MAGNETS
}

\author{
M. A. Green ${ }^{1}$, E. H. Hoyer ${ }^{1}$, R. D. Schlueter ${ }^{1}$, C. E. Taylor ${ }^{1}$, S. T. Wang ${ }^{2}$ \\ and J. Zbasnik ${ }^{1}$ \\ 1. E. O. Lawrence Berkeley National Laboratory \\ University of California \\ Berkeley, CA 94720, USA \\ 2. Wang NMR Incorporated \\ Livermore CA 94550, USA
}

\begin{abstract}
The $1.9 \mathrm{GeV}$ Advance Light Source (ALS) at the Lawrence Berkeley National Laboratory (LBNL) produces photons with a critical energy of about $3.1 \mathrm{keV}$ at each of its thirty-six $1.3 \mathrm{~T}$ gradient bending magnets. It is proposed that at three locations around the ring the conventional gradient bending magnets be replaced with superconducting bending magnets with a maximum field of 5.6 T. At the point where the photons are extracted, their critical energy will be about $12 \mathrm{keV}$. In the beam lines where the SuperBend superconducting magnets are installed, the $\mathrm{X}$ ray brightness at $20 \mathrm{keV}$ will be increased over two orders of magnitude. This report describes three different refrigeration options for cooling the three SuperBend dipoles. The cooling options include: 1) liquid helium and liquid nitrogen cryogen cooling using stored liquids, 2) a central helium refrigerator (capacity 70 to $100 \mathrm{~W}$ ) cooling all of the SuperBend magnets, 3) a Gifford McMahon (GM) cryocooler on each of the dipoles. This paper describes the technical and economic reasons for selecting a small GM cryocooler as the method for cooling the SuperBend dipoles on the LBNL Advanced Light Source.
\end{abstract}

\section{INTRODUCTION}

The ALS at LBNL is a national user facility for vacuum ultraviolet ( $6 \mathrm{eV}$ to $6 \mathrm{keV})$ and soft $x$ rays $(>6 \mathrm{keV})$ synchrotron radiation. The $1.9 \mathrm{GeV}$ ALS electron storage ring has a circumference of about 200 meters. The ALS ring consists of twelve cells each with three combined function $\mathrm{C}$ shaped $1.3 \mathrm{~T}$ bending magnets (with the return flux leg pointing to the inside of the ring) about one meter long. Each dipole generates photons with a critical energy of $3.1 \mathrm{keV}$ when the electron beam energy is $1.9 \mathrm{GeV}$. These photons can be delivered to users through forty-eight ports around the ring to users outside of the synchrotron shielding. Each ring cell contains two defocusing quadrupoles, a long straight section with two pairs of quadrupoles (for insertion devices and RF), and four correction sextupoles. The conventional ALS electron storage ring has a periodicity of twelve. The twelve cell lattice configuration and the relatively low energy of the electron beam both contribute to the small beam size in the machine. The small electron beam size results in the creation of bright photon beams for current ALS users in the energy range from $1 \mathrm{keV}$ to $5 \mathrm{keV}$. 
Some years ago, a study was commissioned on ways to increase the photon energy from the ALS1. One approach that was suggested was to increase the energy of the photons from the ring by increasing the bending field in the ring dipoles. (The critical energy of the synchrotron radiation photons generated by a storage ring dipole is proportional to the dipole induction and the electron energy squared.) The photon energy in selected ports can be increased by increasing the dipole induction in selected places around the ring. A portion of the ring dipoles can be replaced with superconducting dipoles with minimum disturbance to the ring lattice and the other magnets in the ring. A number of test SuperBend test coils were built and tested. The fourth SuperBend coil was successful in that it did not train and could be charged to the its full design field in less than 100 seconds, 2 .3. Once a successful test magnet with a suitable magnetic field was made, the SuperBend project could move forward.

Adding superconducting dipoles to the existing ALS lattice will change its periodicity (at least to second order). From a beam dynamics standpoint, the number of dipoles that can be changed is three, four, six or twelve. All of the studies concluded that the center dipole of the three dipoles in the cell was the one that should be changed. The addition of superconducting dipoles to the ALS lattice will increase the beam emittance due to the rebound effect of more energetic photons being generated by the higher field dipole. Since ALS has a dedicated user community in the $1 \mathrm{keV}$ to $5 \mathrm{keV}$ range, it was essential that the increase in emmittance be minimized. As a result, the higher field superconducting dipoles are put in only three locations around the ring.

It has been clear from the beginning that the superconducting dipoles must fit in the existing ALS ring with minimum disturbance to the users and existing ring components. The SuperBend superconducting dipoles must have the following characteristics 1) The dipole must provide $1.106 \mathrm{~T} \mathrm{~m}$ of bending when the stored electron energy is $1.9 \mathrm{GeV}$. (The beam must be bent 10 degrees.) 2) The dipole must be a $\mathrm{C}$ shaped dipole with the opening of the $\mathrm{C}$ facing away from the ring center. The magnetic field must be generated in a warm bore that fits around the existing ALS vacuum chamber. 3) The magnetic induction in the region where the extracted $x$ rays are produced should be greater than $5 \mathrm{~T}$ so that the photons will have a critical energy near $12 \mathrm{keV} 4$ ) The superconducting dipole must increase its magnetic field as the ALS main ring beam energy increases from 1.5 to $1.9 \mathrm{GeV}$. The charge time from $1.5 \mathrm{GeV}$ to $1.9 \mathrm{GeV}$ must be the same as for the conventional magnet system, 100 seconds. 5) The superconducting dipoles must be operated powered at all times, so that their field can be adjusted along with the rest of the ring magnets. Persistent operation is feasible but not desirable. 6) Superconducting dipole quenches must be avoided during normal machine operation, in order retain high levels of ALS availability. A power failure must not cause the superconducting magnets to quench.

\section{REFRIGERATION REQUIREMENTS FOR THE SUPERBEND DIPOLES}

The superconducting dipole has a $\mathrm{C}$ shaped iron yoke that is $380 \mathrm{~mm}$ long, in the direction of the beam. The height of the iron yoke is $820 \mathrm{~mm}$; the width of the iron yoke is $700 \mathrm{~mm}$. The gap between the poles on the yoke is $235 \mathrm{~mm}$. Within this gap is a pair of race track shaped superconducting coils that are $67 \mathrm{~mm}$ thick. Between the race track shaped superconducting coils, there is a $100 \mathrm{~mm}$ gap that contains superinsulation, gap shields, the cryostat Vacuum chamber walls, the ALS vacuum chamber and clearance for assembly and adjustment. The magnet system cold mass is about $1680 \mathrm{~kg}$.

The cryostat is $1474 \mathrm{~mm}$ high, $902 \mathrm{~mm}$ wide and $648 \mathrm{~mm}$ thick in the beam direction. The center line of the magnet gap is $506 \mathrm{~mm}$ from the bottom of the magnet cryostat vessel. The warm gap in the SuperBend dipole is $54.9 \mathrm{~mm}$. The ALS vacuum chamber thickness is $51.6 \mathrm{~mm}$, which gives a total clearance of $\pm 1.65 \mathrm{~mm}$ between the SuperBend magnet cryostat opening and the ALS vacuum chamber. Within the cryostat envelope the magnet cold mass, a liquid helium vessel, a liquid nitrogen vessel, and the cold parts of a $4 \mathrm{~K}$ refrigeration system must reside.

The SuperBend dipole magnet cooling requirements are drven by the following considerations: 1) The maximum operating temperature for the magnets is $4.5 \mathrm{~K}$. 2) The heat load in the $4 \mathrm{~K}$ part of the cryostat is less than 1 watt. 3) The magnet cold mass is about $1680 \mathrm{~kg}$. Liquid cryogens can be used to cool the magnet system cold mass. 4) The superconducting coils are indirectly cooled. They are cooled by conduction from the iron yoke and through a copper strap from the helium vessel. The yoke is in turn cooled by 
conduction from a tank filled with liquid helium. 5) The superconducting magnet coils will be powered at all times. The electrical leads from $300 \mathrm{~K}$ to $4 \mathrm{~K}$ will be a factor in defining the refrigeration needed for the SuperBend magnet system. 6) The maximum magnet charge rate is 3 A per second, which means that the magnet is charged to its full design field in 100 seconds. 7) The magnet is cycled between $4.4 \mathrm{~T}$ and $5.6 \mathrm{~T}$ at about $0.06 \mathrm{~T}$ per second for injection and accelleration. Then the dipole field stays constant at $5.6 \mathrm{~T}$ for nearly four hours. 8) The cooling system must be very reliable, since the SuperBend magnets are integral part of the ALS storage ring. 9) Cryogenic liquid cooling as a back up for mechanical refrigeration is acceptable. 10) Magnet cooling must not be affected by power shut downs of an hour or less.

\section{Table 1 Estimated Parasitic Heat Loads for a SuperBend Magnet}

Heat Load Source

Parasitic Heat Loads at 50 to $80 \mathrm{~K}$ from $300 \mathrm{~K}$

St. St. Tubes for He Vent, LN Tank Support \& Cool Down

Radiation and Conduction through the MLI

Eight Cold Mass Suspension Bands

Instrumentation Wires

Total 50 to $80 \mathrm{~K}$ Heat Load from $300 \mathrm{~K}$

Parasitic Heat loads at $4.0 \mathrm{~K}$ from a $50 \mathrm{~K}$ shield and Intercepts

Thin Wall St. St. Neck Tubes and Cool Down Tubes

Eight Cold Mass Suspension Bands

Radiation and Conduction Through the MLI

Twenty $0.13 \mathrm{~mm}$ Dia. Instrumentation Wires from $300 \mathrm{~K}$

Total $4.0 \mathrm{~K}$ Heat Load from $50 \mathrm{~K}$

Parasitic Heat loads at $4.0 \mathrm{~K}$ from a $80 \mathrm{~K}$ shield and Intercepts

Thin Wall St. St. Neck Tubes and Cool Down Tubes

Eight Cold Mass Suspension Bands

Radiation and Conduction Through the MLI

Twenty $0.13 \mathrm{~mm}$ Dia. Instrumentation Wires from $300 \mathrm{~K}$

Total $4.0 \mathrm{~K}$ Heat Load from $50 \mathrm{~K}$
Heat Load Magnitude

The estimated parasitic heat loads at $4 \mathrm{~K}$ and at an intermediate shield and intercept temperature between 50 and $80 \mathrm{~K}$ is presented in Table 1 . The parasitic heat leak to the shields and intercepts is of the order of 8 to $10 \mathrm{~W}$. Heat leak due to magnet current leads may be added to the parasitic heat leak at 50 to $80 \mathrm{~K}$. The heat leak into the $4 \mathrm{~K}$ region is strongly dependent on the temperature of the shields and intercepts. It is quite reasonable to expect this heat will be smaller than $0.5 \mathrm{~W}$ even when the shields operate with liquid nitrogen as a coolant. Care must be taken to ensure that the heat leak at $4 \mathrm{~K}$ is below 0.3 to $0.5 \mathrm{~W}$. In all cases, there will be additional heat leaks at $4 \mathrm{~K}$ due to the 320 A magnet electrical leads. The type of leads used to carry current into the magnet has a strong effect on the $4 \mathrm{~K}$ refrigeration needed to cool the SuperBend magnet system.

\section{COOLING OPTIONS FOR THE SUPERBEND DIPOLES}

Three cooling options were investigated for the SuperBend magnet system. In all cases, the liquid helium reservoir is assumed to hold 91 liters and the liquid nitrogen reservoir is assumed to hold 60 liters. The three cooling option studied are as follows: 1) Liquid nitrogen and liquid helium cooling from dewars was considered. The LBNL team looked at cases with high Tc Superconductor (HTS) leads and conventional gas cooled copper leads cooled with the boil off gas from the helium dewar. 2) A $4.4 \mathrm{~K}$ central refrigerator was looked at to cool the three magnets. HTS lead and standard gas cooled leads were looked at for this option as well. 3) Individual cryocoolers for each magnet was studied. The type of cryocooler used depends strongly on whether conduction cooled or gas cooled leads are used on the magnets.

Stored liquid cryogen cooled systems were studied with and without a $30 \mathrm{~K}$ shield between the liquid nitrogen $(80 \mathrm{~K})$ shield and the $4.3 \mathrm{~K}$ region. This shield was cooled with a small amount of boil off helium from the $4.3 \mathrm{~K}$ region. The addition of a $30 \mathrm{~K}$ shield 
reduces the heat load at $4.3 \mathrm{~K}$ by a factor of three, if the magnet is in persistent mode with the leads retracted. Since this is not an option for SuperBend the effect of the shield is not so dramatic. With gas cooled leads, the boil off is not changed when a $30 \mathrm{~K}$ shield is used. When $320 \mathrm{~A}$ gas cooled leads are used, the helium boil off is 1.16 liters per hour and the liquid nitrogen boil off is 0.19 liters per hour. HTS leads will reduce the helium boil off to 0.70 liters per hour without a $30 \mathrm{~K}$ shield and 0.42 liters per hour with a $30 \mathrm{~K}$ shield, but they will increase the nitrogen boil off to 0.82 liters per hour because the heat leak at $80 \mathrm{~K}$ increases due to heat leak down the 320 A solid copper electrical leads from $300 \mathrm{~K}$.

The helium dewars must be filled every three to eight days depending on the type of lead and whether or not a gas cooled shields are used between the $80 \mathrm{~K}$ shield and the $4.3 \mathrm{~K}$ region. The nitrogen dewar can be put on an automatic fill system that keeps the nitrogen dewar full. With liquid helium priced at $\$ 3.00$ per liter and liquid nitrogen priced at $\$ 0.25$ per liter and a transfer efficiency of 80 percent, the cryogen cost for ten years would be from $500 \mathrm{k} \$$ to $1160 \mathrm{k} \$$ depending on the type of leads used. Added to the ten year operating cost is the cost of the transfer line system, the cost of HTS leads and the ten years cost of the technician that keeps the dewars full. The estimated ten year refrigeration cost for three SuperBend magnets using liquid cryogen ranges from $630 \mathrm{k} \$$ to $1280 \mathrm{k} \$$ depending on the type of leads used. A schematic of a liquid cryogen cooled SuperBend magnet is shown in Figure 1a.

The central refrigeration system studied assumed that a single $70 \mathrm{~W}$ Claude cycle refrigerator is used to cool all three SuperBend magnets. Cooling at $80 \mathrm{~K}$ is assumed to be provided by liquid nitrogen. The refrigeration required per each SuperBend magnet is dependent on the type of leads used on the magnet. For $320 \mathrm{~A}$ gas cooled leads, $4.7 \mathrm{~W}$ of refrigeration at $4.4 \mathrm{~K}$ is required. If HTS leads are used, the per magnet refrigeration requirement goes down to 0.4 to $0.6 \mathrm{~W}$. The cooling needed to cool the three SuperBend magnets using a central refrigerator is dominated by the transfer line heat leak from the refrigerator to the magnets. The minimum length of transfer line to the magnets to and from the refrigerator is about 360 meters (from and back to the refrigerator). Shielded transfer lines in a common vacuum can achieve heat leaks of $0.1 \mathrm{~W}$ per running meter at $4.4 \mathrm{~K}$. A single 70.W refrigerator can provide cooling to up to six SuperBend magnets operating with gas cooled leads or more than a dozen SuperBend magnets operating with HTS leads.

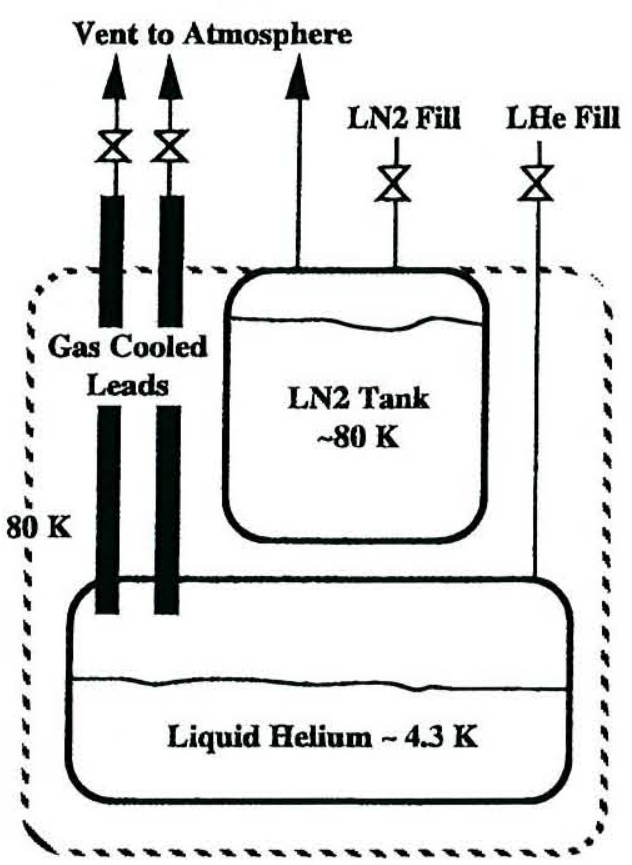

a) Stored Liquid Cooling

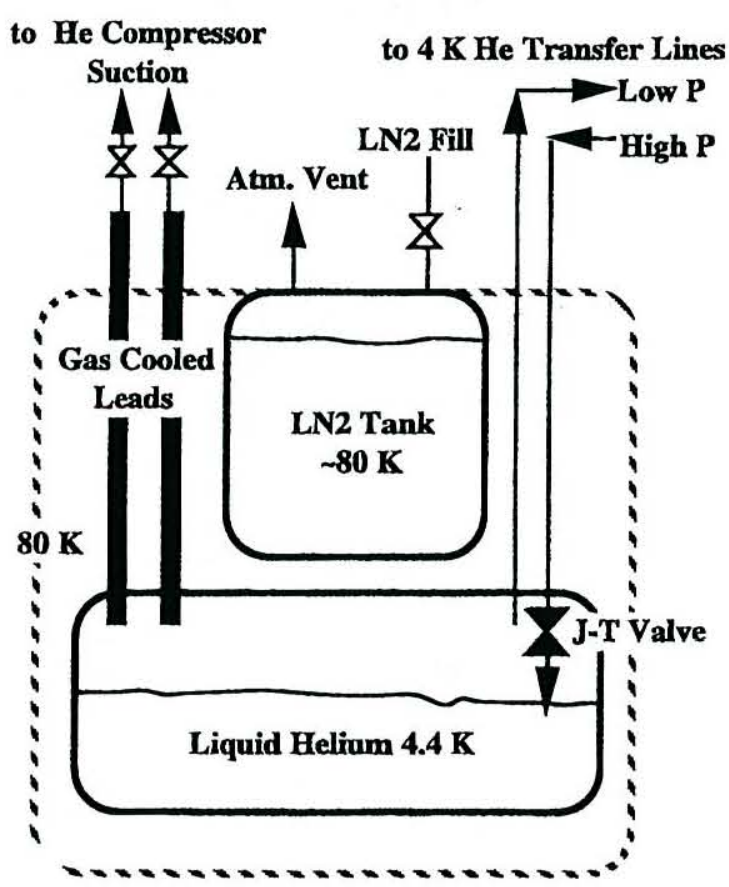

b) Central Refrigerator Cooling

Fig. 1 Schematic Representations of Stored Cryogen Cooling and Cooling with a Central Refrigerator for SuperBend Magnets with Gas Cooled Leads 
In the event of a refrigerator failure, the magnets can be operated using stored liquid cryogens until the refrigerator is repaired. The transfer line system has to include a provision for precooling the lines before they can be reconnected to the magnets. The capital cost of the refrigerator (based on a PSI Model 1400) plus the around the ring transfer lines is about $450 \mathrm{k} \$$ plus an additional $20 \mathrm{k} \$$ per magnet $t^{4}$. The ten year operating cost for liquid nitrogen (at $\$ 0.25$ per liter) plus electric power at $\$ 0.12$ per $\mathrm{kWh}$ is about $\$ 670 \mathrm{k} \$$ plus $20 \mathrm{k} \$$ per magnet. The capital cost plus ten year operating cost for three SuperBend magnets is about $1240 \mathrm{k} \$$. For twelve SuperBend magnets with HTS leads, the capital plus ten year operating cost goes up to about $1600 \mathrm{k} \$$. The capital cost of the refrigeration system plus the ten year cost of cooling cost for six SuperBend magnet with gas cooled leads would be about 1460 $\mathrm{k} \$$. A schematic representation of cooling a SuperBend magnet with gas cooled leads using a central refrigerator is shown in Figure $1 \mathrm{~b}$.

Small cryocoolers can provide refrigeration to cool the shields (at $50 \mathrm{~K}$ ) along with refrigeration at $4 \mathrm{~K}$ to cool the superconducting magnet. The SuperBend group looked at pure Gifford McMahon (GM) cryocoolers and GM cryocooler that use an additional heat exchanger and a J-T valve to achieve temperatures in $4 \mathrm{~K}$ range. The advantages of a GM cooler with a J-T circuit are the cooler can provide cold gas for conventional gas cooled leads and the temperature at the $4 \mathrm{~K}$ end of the cryocooler is relatively constant with the applied heat load. Pure GM cryocoolers can not provide cold helium to gas cooled leads and the low end temperature changes with the load. The primary advantages of the pure GM cryocoolers are greater reliability and lower cost. The technology that makes the use of pure GM cryocoolers useful for continuously powered superconducting magnets such as SuperBend is low heat leak HTS current leads between the first and second stages of the cryocooler. Without HTS current leads, continuous current excitation of the SuperBend magnet is not possible when it is cooled using a pure GM cryocooler. When a pure GM cryocooler is used with HTS leads, the $4 \mathrm{~K}$ heat leak into the magnets is between $0.3 \mathrm{~W}$ and $0.5 \mathrm{~W}$. Because one is using HTS leads, the heat load at $50 \mathrm{~K}$ is about $40 \mathrm{~W}$. It is clear that for SuperBend, the cryocooler must be sized for refrigeration at $50 \mathrm{~K}$ as well as refrigeration at $4 \mathrm{~K}$. As a result, the SuperBend design team selected a Sumitomo GM 1.5 W (at $4.2 \mathrm{~K}$ ) cryocooler for the SuperBend test magnet.

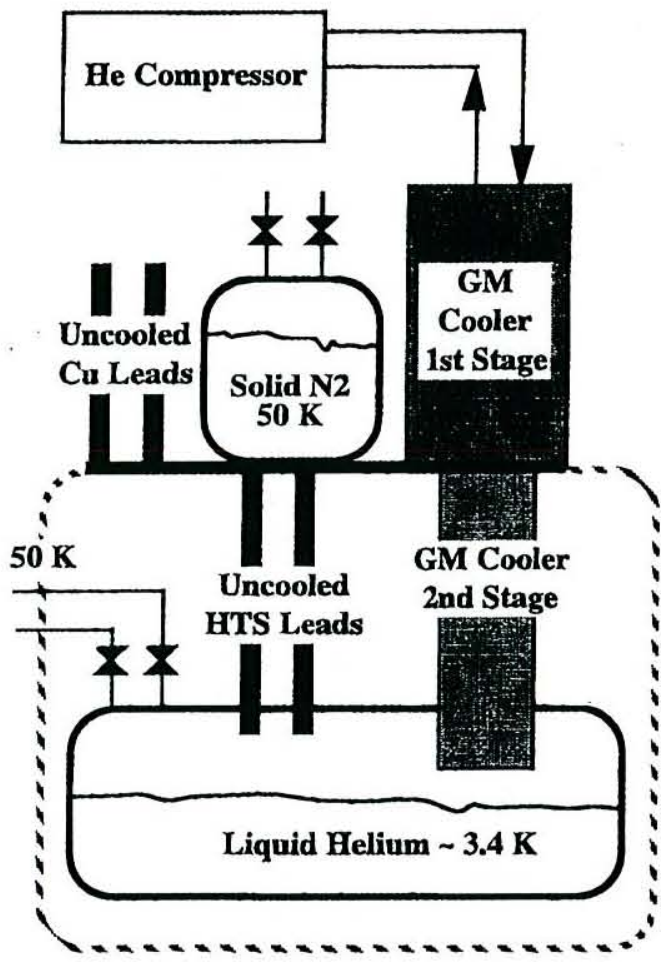

a) GM Cryocooler On with HTS Leads

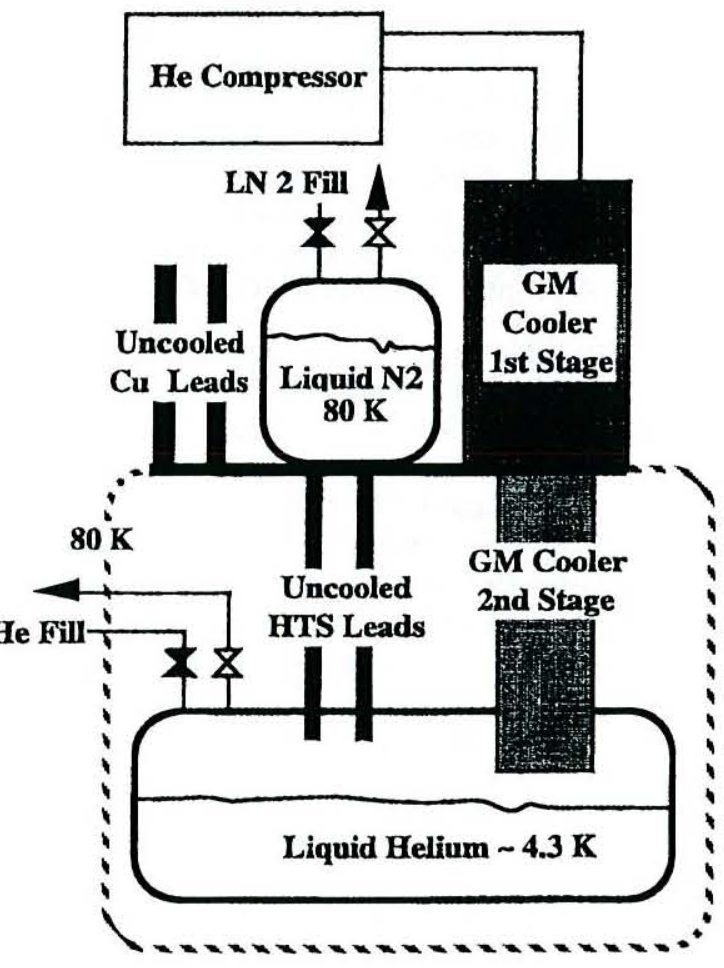

b) GM Cryocooler Off with HTS Leads

Fig. 2 Schematic Representation of Cooling with a Small GM Cryocooler with the Cryocooler On and Off 
The cost of HTS current leads and a pure GM cryocooler suitable for cooling the SuperBend magnet is about $50 \mathrm{k} \$$ per magnet. The ten year cost of operation is about $75 \mathrm{k} \$$ per magnet. The projected capital cost plus the cost of operation for ten years for three SuperBend magnets is $375 \mathrm{k} \$$. The use of small cryocoolers to cool SuperBend magnets is clearly justified economically. Figure 2a shows a schematic representation of SuperBend cooling with a small GM cryocooler. The magnet system shown in Figure 2 is powered through HTS leads.

\section{A GM CRYOCOOLER SYSTEM FOR THE SUPERBEND DIPOLES}

A GM cryocooler can provide cooling at $4 \mathrm{~K}$ to the SuperBend superconducting magnet and $50 \mathrm{~K}$ cooling to the shields and intercepts without any stored cryogens. Since reliability is a key issue for the ALS, back-up cooling for SuperBend using stored liquid cryogens has been selected. This means that the second stage of the cryocooler must provide cooling to a tank of liquid helium as well as the cold mass of the magnet. In addition, the first stage of the cryocooler must cool a tank for liquid nitrogen as well as the shields. Figure 2 illustrates cooling with a cryocooler and back-up cooling with liquid cryogens. The additional cryogen storage tanks do not contribute to the heat leaks into the SuperBend magnet, but the stored cryogen tanks do add to the cost and create safety hazards associated with stored cryogens. The added reliability of having stored cryogens as a back-up for the cryocooler is considered to be worth the extra expense. The heat leaks and estimated boil off rates for stored cryogens for the SuperBend magnet cooled with a $1.5 \mathrm{~W}$ Sumitomo Cryocooler is shown in Table 2 Figure 3 shows a cross-section view of the SuperBend dipole. Shown in Figure 3 are the superconducting coils, the magnet iron, the two cryogen tanks and the cryocooler.

Table 2 SuperBend Dipole Heat Loads with the Cryocooler On and Heat Loads and Liquid Cryogen Boil Off Rates with the Cryocooler Off

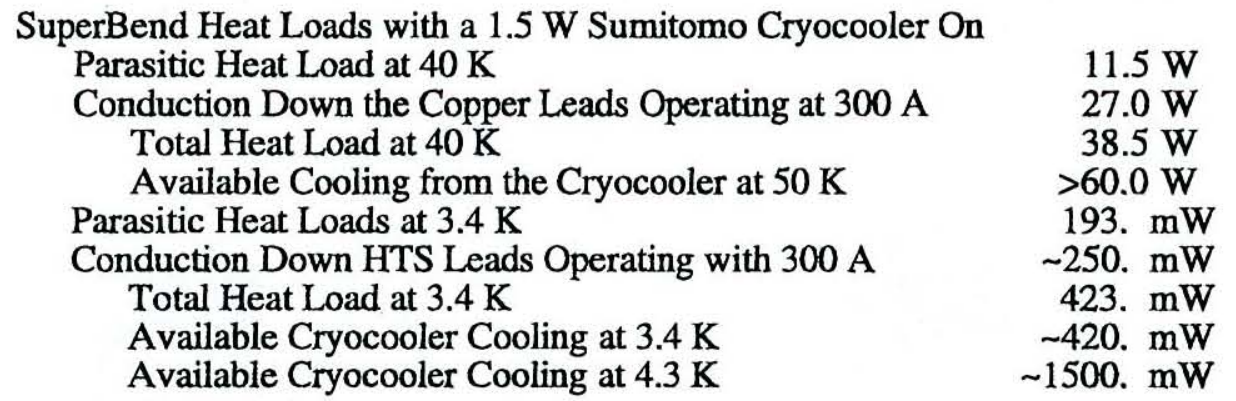

SuperBend Heat Loads and Cryogen Boil Off with the Cryocooler Off

Parasitic Heat Load at $80 \mathrm{~K}$

Conduction Down the Copper Leads Operating at 300 A

Conduction Down Cryocooler First Stage

Total Heat Load at $80 \mathrm{~K}$

Liquid Nitrogen Boil Off

Parasitic Heat Leak at $4.3 \mathrm{~K}$

Conduction Down HTS Leads Operating at $300 \mathrm{~A}$

Conduction Down the Cryocooler Second Stage

Total Heat Load at $4.3 \mathrm{~K}$

Liquid Helium Boil Off

$$
\begin{gathered}
11.5 \mathrm{~W} \\
27.0 \mathrm{~W} \\
\sim 50.0 \mathrm{~W}^{*} \\
\sim 87.5 \mathrm{~W} \\
\sim 2.0 \mathrm{l} \mathrm{per} \mathrm{hr} \\
366 . \mathrm{mW} \\
\sim 400 . \mathrm{mW} \\
\sim 1960 . \mathrm{mW}^{*} \\
\sim 2726 . \mathrm{mW} \\
\sim 4.0 \mathrm{l} \text { per hr }
\end{gathered}
$$

* Sumitomo estimate for the second stage; LBNL estimate for the first stage.

From Table 2, it appears that the use of liquid cryogens is viable back-up cooling method for a SuperBend dipole that is cooled with a pure GM cryocooler. A test cryostat has been set up by the SuperBend magnet group to measure the performance of the Sumitomo $1.5 \mathrm{~W}$ cryocooler operating at various heat loads 5 . The test cryostat also permits one to test the operation of HTS leads between the first and second stages of the cryocooler. The test cryostat will provide an accurate sestimate of the heat load into the magnet vessels when the cryocooler has been shut off. 


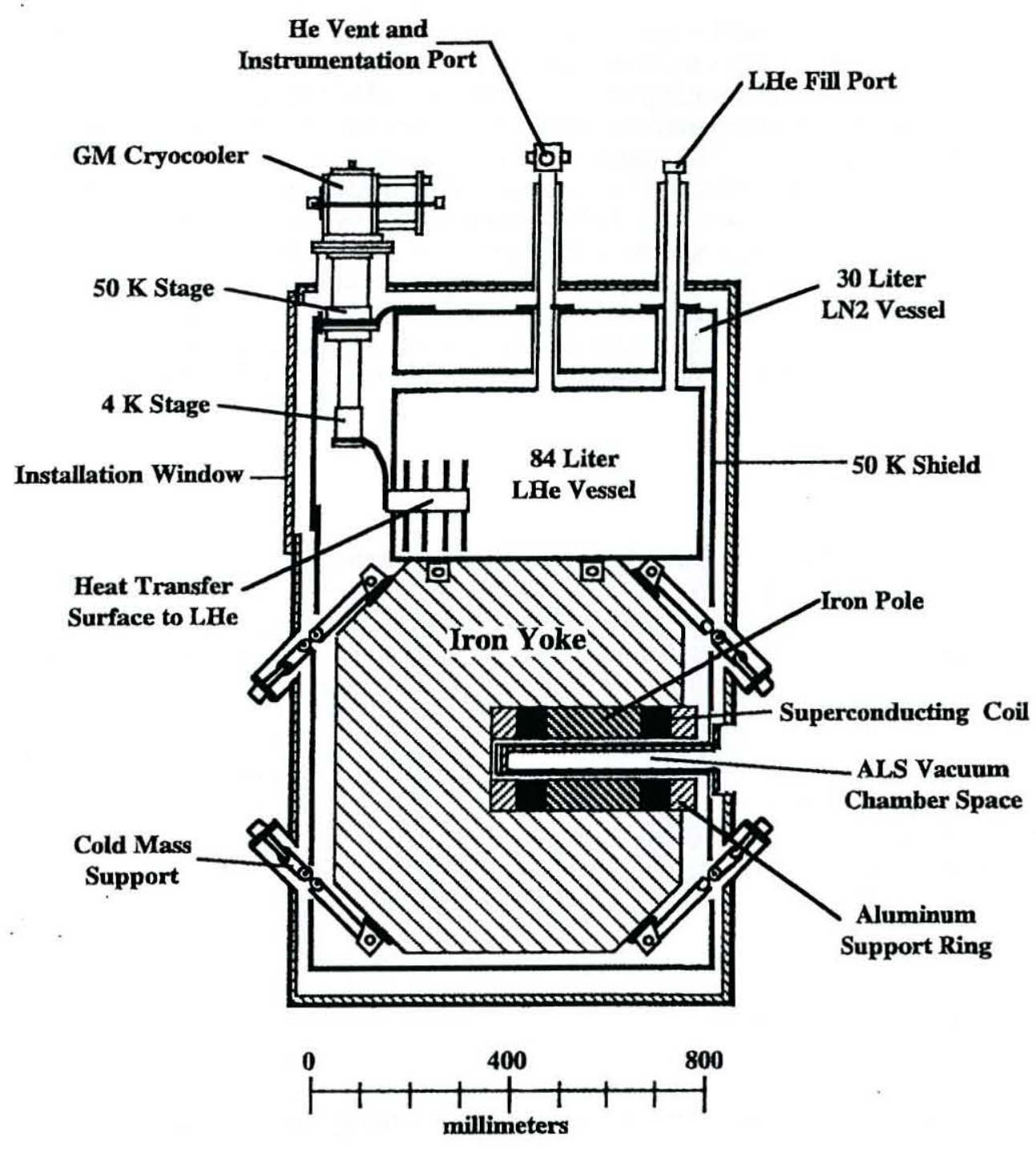

Fig. 3 A Cross-section view of the SuperBend Dipole in a Plane Perpendicular to the ALS Beam

Figure 3 does not show the HTS leads. The leads will be mounted on either side of the cryocooler. The lower end of the HTS leads will be electrically insulated from but thermally connected to the liquid helium tank. The upper end of the HTS leads will be electrically insulated from but thermally connected to the liquid nitrogen tank. Niobium Titanium will connect the lower end of the HTS leads to the superconducting coils. The niobium titanium leads will be cooled by conduction (through an electrical insulator) to the magnet case. The temperature margin for the niobium titanium leads should be at least $3 \mathrm{~K}$

Figure 3 also does not show how the SuperBend superconducting is to be cooled down from room temperature $(300 \mathrm{~K})$ to its normal operating temperature $3.4 \mathrm{~K}$. The Sumitomo cryocooler is capable of cooling down SuperBend from room temperature to $3.4 \mathrm{~K}$. The time to cool down the magnet to $80 \mathrm{~K}$ using the cryocooler alone is about 500 hours. An additional 100 hours is needed to bring the magnet temperature down to $4 \mathrm{~K}$. It is clear that the magnet cool down should be done using liquid nitrogen and liquid helium. A liquid cryogen cooling circuit mounted on copper plates that will encase the iron yoke will allow liquid helium and liquid nitrogen to be used to cool down the SuperBend dipole. The use of liquid cryogens flowing through these tubes should speed up the magnet cool down be at least a factor of twenty.

A number of fault modes were studied. The results of the fault mode study are as follows: 1) The failure of a cryocooler compressor does not affect the operation of the SuperBend dipole. The compressor can replaced quickly since the plumbing between the 
compressor and the cryocooler is designed so that the compressor can be disconnected from the cryocooler while the cryocooler heads are cold. 2) Cryocooler head failure is a rare occurrence, liquid cryogen cooling will keep the SuperBend magnets operating until the cryocooler head can be changed during a scheduled shutdown. 3) An electrical power failure will shut off the cryocooler. The magnet is kept cold using stored cryogens. Once power has been restored the cryocooler can be restarted. 4) A magnet quench should not affect the cryocooler, but the magnet will have to be cooled back down using liquid cryogens. The cryocooler can run while this cool down is taking place. 5) A high Tc superconducting lead turning normal should be a rare event that is indicative of some other fault in the system. A lead turning normal requires that the magnet be discharged to protect the HTS lead. Depending on the discharge time required to protect the lead, the magnet may or may not quench during the discharge. Once the magnet is cold, one should be able to repower the magnet.

\section{CONCLUSION}

Small GM cryocoolers appear to be the most cost effective cooling system for the three SuperBend dipoles for the ALS. A centralized refrigeration system with transfer lines around the ALS ring does not become competitive until the number of SuperBend dipoles exceeds twelve. GM cryocoolers can provide refrigeration at $50 \mathrm{~K}$ and $4 \mathrm{~K}$ simultaneously. Extra transfer lines are required for a centralized refrigeration system to do this. The use of high HTS current leads between $50 \mathrm{~K}$ and $4 \mathrm{~K}$ permits the SuperBend magnets to be powered continuously. The size of the GM cryocooler is dictated by the cooling requirements at $50 \mathrm{~K}$ not the cooling requirements at $4 \mathrm{~K}$. The primary heat load at $50 \mathrm{~K}$ is the heat leak down the copper $300 \mathrm{~A}$ lead from $300 \mathrm{~K}$ to $50 \mathrm{~K}$.

In an emergency, the SuperBend magnets can be kept cold using stored liquid helium and liquid nitrogen. The HTS leads must be capable of carrying up to $320 \mathrm{~A}$ while their upper end is at $80 \mathrm{~K}$. The use of small GM cryocoolers is justified in terms of reliability as well as çost.

\section{ACKNOWLEDGMENTS}

This work was supported by the Director of the Office of Basic Energy Science, High Energy Physics Division, United States Department of Energy under contract number DEAC03-76SF00098.

\section{REFERENCES}

1. C. E. Taylor and S. Caspi, "A. 6.3 T Bend Magnet for the Advanced Light Source," IEEE Trans. Magnetics 32, No 4, (1996)

2. C. E. Taylor, et al, "Test of a High-Field Bend Magnet for the ALS," to be published in IEEE Trans. Appl. Superconductivity 9, No. 2 (1999)

3. A. Lietzke, "SuperBend 4 Magnetic Measurements," LBNL Superconducting Magnet Group Internal Report SC-MAG 633, September 1998

4. M. A. green and R. Byrns, "An Update on Estimating the Cost of Cryogenic Refrigeration," Advances in Cryogenic Engineering 43, p 1661, Plenum Press, (1997)

5. J. Zbasnik, et al, "Tests of a GM Cryocooler and High Tc Leads for Use on the ALS SuperBend Magnets," presented at the 1999 Cryogenic Engineering Conference, Montreal Quebec, Canada, 13 to 16 July 1999, This Proceedings 